\title{
MEAN FIELD DYNAMO IN THICK DISKS AROUND KERR BLACK HOLES: HIGH ORDER AXISYMMETRIC SIMULATIONS*
}

\author{
M. BUGLI \\ Max-Planck-Institute für Astrophysik \\ Karl-Schwartzschild Strasse 1, 85741 Garching, Germany \\ matteo@mpa-garching.mpg.de \\ L. DEL ZANNA \\ Dipartimento di Fisica e Astronomia, Università di Firenze \\ Via Sansone 1, 50019 Sesto Fiorentino (FI), Italy \\ N. BUCCIANTINI \\ INAF - Osservatorio Astrofisico di Arcetri \\ L.go E. Fermi 5, 50125 Firenze, Italy
}

Received 30 October 2013

Published 21 March 2014

\begin{abstract}
We present here a kinematic study of dynamo effects in thick accretion disks using the most recent version of the ECHO code. This allowed us to perform high-order simulations of a resistive plasma within the $3+1$ GRMHD frame with a fully covariant mean-field dynamo closure for the Ohm's law. The magnetic field goes through an exponential growth within few orbital periods of the disk, and we derive butterfly diagrams qualitatively in the same fashion as in the Sun. Finally, a typical time-scale of the field evolution is found, that depends on the dynamo and resistivity parameters and that could be related to the periodicities observed in several accreting systems.
\end{abstract}

Keywords: Magnetic fields; relativity; magnetohydrodynamics; accretion; accretion disks.

PACS Numbers: 97.10.Gz, 98.62.Mw, 47.65.Md

\section{Introduction}

Ordered, large-scale magnetic fields are believed to be a fundamental ingredient of the accretion processes that power most of the astrophysical sources of highenergy emission, like active galactic nuclei or gamma ray bursts. Their role in the formation of relativistic jests is crucial, but it is still not clear how such magnetic

*This is an Open Access article published by World Scientific Publishing Company. It is distributed under the terms of the Creative Commons Attribution 3.0 (CC-BY) License. Further distribution of this work is permitted, provided the original work is properly cited. 
fields originate. A solution could come from a dynamo process, and if we consider the presence of small-scale correlated fluctuations in the flow due to turbulence (that invariably arises in astrophysical plasmas with very high fluid and magnetic Reynolds numbers) a mean-field dynamo mechanism ${ }^{1}$ could lead to a large-scale effective electromotive force capable to continuously amplify and generate a largescale magnetic field. The first formulation of a fully covariant closure of the GRMHD equations for a non-ideal plasma with a mean-field dynamo mechanism has been given by Ref. 2, but so far it has never been applied to an actual astrophysical system. Here we present the first example (to our knowledge) of a resistive GRMHD high order simulation of a thick disk orbiting around a Kerr black hole with a meanfield dynamo mechanism. Given a small magnetic seed, we follow the evolution of the fields trough the kinematic phase, assuming therefore that their feedback on the other quantities can still be neglected.

\section{Kinematic Dynamo Model}

For classical MHD, the introduction of a mean-field mechanism leads to an additional term in the classic resistive Ohm's law, so that ${ }^{1}$

$$
\boldsymbol{E}^{\prime}=\boldsymbol{E}+\boldsymbol{v} \times \boldsymbol{B}=\eta \boldsymbol{J}+\xi \boldsymbol{B},
$$

where $\boldsymbol{E}, \boldsymbol{B}$ and $\boldsymbol{J}$ are respectively the electric field, magnetic field and current measured by an eulerian observer, $\boldsymbol{E}^{\prime}$ is the electric field measured in the frame comoving with the fluid-velocity $\boldsymbol{v}, \eta$ is the magnetic resistivity due mostly to meanfield effects (and in smaller part to collisions), $\xi$ is the mean-field dynamo coefficient (most commonly employed in the literature as $\alpha \equiv-\xi$ ) and we have set $c \rightarrow 1,4 \pi \rightarrow$ 1 . The $\xi$-term is a direct result of the assumption of correlated turbulent motions in the plasma, and its action combined with the differential rotation of a system such an accretion disk might lead to a so called $\alpha \Omega$ dynamo. In the kinematic case, the dynamics of such a system is characterized by the following dynamo numbers:

$$
C_{\xi}=\xi R / \eta, \quad C_{\Omega}=\Delta \Omega R^{2} / \eta,
$$

which estimate the efficiency of the dynamo processes (due respectively to the meanfield dynamo and the differential rotation) in enhancing the magnetic field amplitude against the dissipation. In this relations $R$ represents the radial (and vertical) length scale of the disk, while $\Delta \Omega$ is the difference in angular velocity between the center and the inner edge of the disk.

A fully covariant form of the Ohm's law for a resistive plasma with a mean-field $\alpha$-dynamo effect is given by Ref. 2 :

$$
e^{\mu}=\eta j^{\mu}+\xi b^{\mu},
$$

where $e^{\mu}, b^{\mu}$ and $j^{\mu}$ are respectively the electric field, magnetic field and current measured by an observer comoving with the fluid 4 -velocity $u^{\mu}$. Since we let only 

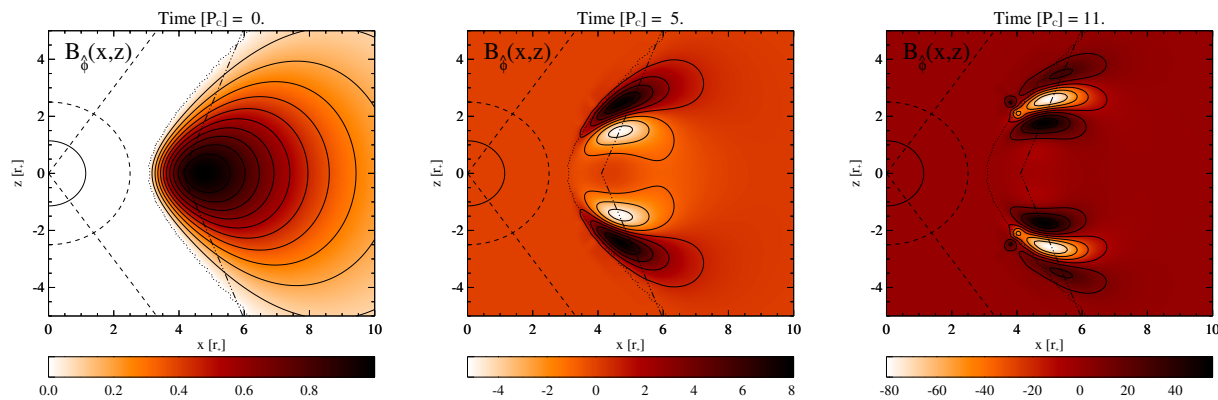

Fig. 1. Evolution of the toroidal component $B_{\hat{\phi}}$ for the Model 1. The dot-dashed lines starting from the center of the disk lie on the maxima of the magnetic field and define the cut for producing the butterfly diagrams.

the electric and magnetic fields evolve through time, we just need to integrate the Maxwell equations, which in the $3+1$ formalism can be written as: ${ }^{2}$

$$
\begin{gathered}
\gamma^{-1 / 2} \partial_{t}\left(\gamma^{1 / 2} \boldsymbol{B}\right)+\boldsymbol{\nabla} \times(\alpha \boldsymbol{E}+\boldsymbol{\beta} \times \boldsymbol{B})=0, \\
\gamma^{-1 / 2} \partial_{t}\left(\gamma^{1 / 2} \boldsymbol{E}\right)-\boldsymbol{\nabla} \times(\alpha \boldsymbol{B}-\boldsymbol{\beta} \times \boldsymbol{E})+(\alpha \boldsymbol{v}-\boldsymbol{\beta}) \boldsymbol{\nabla} \cdot \boldsymbol{E} \\
=-\alpha \Gamma \eta^{-1}\{[\boldsymbol{E}+\boldsymbol{v} \times \boldsymbol{B}-(\boldsymbol{E} \cdot \boldsymbol{v}) \boldsymbol{v}]+\xi[\boldsymbol{B}-\boldsymbol{v} \times \boldsymbol{E}-(\boldsymbol{B} \cdot \boldsymbol{v}) \boldsymbol{v}]\},
\end{gathered}
$$

where $\gamma$ is the determinant of the spatial metric tensor, $\alpha$ is the lapse function, $\boldsymbol{\beta}$ is the shift vector and $\Gamma \equiv\left(1-v^{2}\right)^{-1 / 2}$ is the Lorentz factor.

\section{Simulation Setup and Results}

As hydrodynamical background we choose a differential rotating unmagnetized thick torus orbiting a Kerr black hole with mass $M_{B H}=1$ and specific angular momentum $a=0.99$, in Boyer-Lindquist coordinates, as described in Ref. 3. Dynamo will select the fastest growing eigenmode, so the geometry of the initial seed magnetic field is not important. We tried either a purely toroidal seed-field or a purely poloidal one. The electric field is then set as $\boldsymbol{E}=-\boldsymbol{v} \times \boldsymbol{B}$.

The resistivity $\eta$ inside the disk scales as $\eta_{\text {disk }} \sqrt{\rho}$, and drops to a much smaller value $\eta_{\text {atm }} \equiv 10^{-5}$ in the atmosphere (quasi-ideal), while $\xi$ is set to zero in the atmosphere and in the disk scales as $\xi_{d i s k} \sqrt{\rho} \cos \theta$ to get the correct symmetrical behavior of the $\alpha \Omega$ dynamo with respect to the equator. Since $\eta$ is usually very small in astrophysical situations, Eq. (5) is stiff involving terms $\propto \eta^{-1}$. For this reason we used for the time integration the 3rd-order IMplicit-EXplicit (IMEX) Runge-Kutta schemes developed by Ref. 4 and used (but at 2nd-order) in resistive special relativistic MHD by Ref. 5, and in general relativity by Ref. 2 .

Starting with a toroidal magnetic field a poloidal component arises due to the $\alpha$-effect, while an initial poloidal field generates, through the $\Omega$-effect, a toroidal component. Both of them grow exponentially with the same rate, and the ratio 

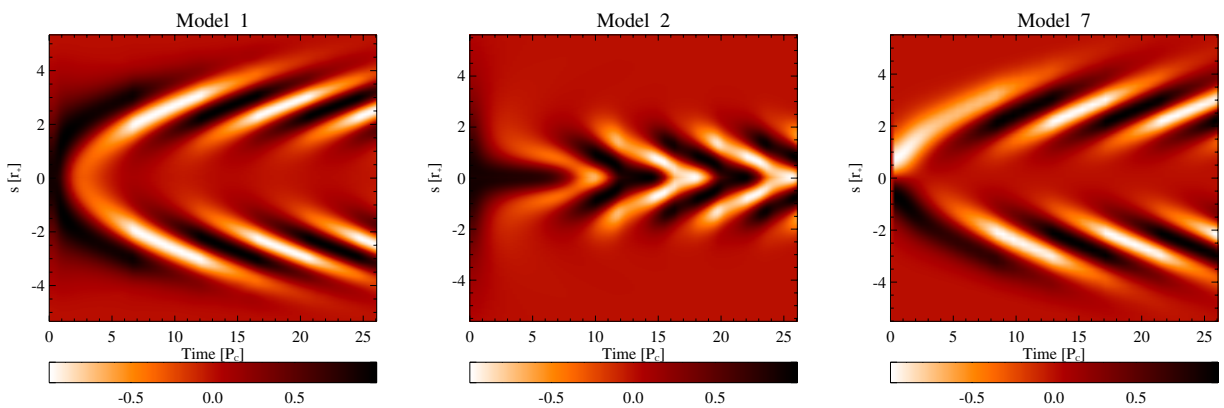

Fig. 2. Butterfly diagrams for the models 1,2 and 7, showing the value of the toroidal magnetic field along the trajectories of the drifting versus the time. The magnetic field has been normalized to its maximum value in the whole domain at every time, which is measured in units of the orbital period of the center of the disk $P_{c}$.

between their maximum values ranges from $4 \%$ to $42 \%$. In every model, after a few orbital periods of the disk center $P_{c} \simeq 76.5 G M_{B H} / c^{3}$, the system reaches an eigenstate with a dynamo wave that propagates towards (away from) the equatorial plane for $\xi<0(\xi>0)$. We produced for each model a butterfly diagram considering the value of the toroidal field along the trajectories of its maxima at different times. From this diagrams is evident the periodicity of the eigenstate and its spatial scale. For larger values of $\xi$ we retrieve smaller periods and smaller spatial scales, while decreasing the action of the mean field dynamo both periods and spatial scales increase. Moreover the particular symmetry of the initial magnetic fields (dipolar or quadrupolar) does not affect the dynamical characteristics of the eigenstate selected by the problem and it is conserved during the evolution of the mode, which is then degenerate in the parity of the magnetic field. The turbulence properties set a time-scale dependent by $\eta$ and $\xi$ unrelated to the larger-scale quantities, that could explain some of the time-variability observed in accreting systems. ${ }^{6}$

Table 1. Results of the study on the $\alpha \Omega$ dynamo.

\begin{tabular}{cccccccrcc}
\hline & $\boldsymbol{B}_{\text {init }}$ & $\eta_{\text {disk }}$ & $\xi_{\text {disk }}$ & $C_{\xi}$ & $C_{\Omega}$ & Growth Rate & Period & $s_{\max }$ & $B_{P} / B_{T}$ \\
\hline Model 1 & $\boldsymbol{B}_{T}$ & $10^{-3}$ & $10^{-3}$ & 5 & 400 & 0.39 & 8.43 & 2.41 & 0.13 \\
Model 2 & $\boldsymbol{B}_{T}$ & $10^{-3}$ & $-10^{-3}$ & -5 & 400 & 0.21 & 8.17 & 0.04 & 0.13 \\
Model 3 & $\boldsymbol{B}_{T}$ & $10^{-3}$ & $5 \times 10^{-3}$ & 25 & 400 & 1.58 & 2.77 & 2.05 & 0.42 \\
Model 4 & $\boldsymbol{B}_{T}$ & $10^{-3}$ & $2 \times 10^{-4}$ & 1 & 400 & 0.07 & 26.38 & 2.63 & 0.04 \\
Model 5 & $\boldsymbol{B}_{T}$ & $5 \times 10^{-3}$ & $5 \times 10^{-3}$ & 5 & 80 & 0.39 & 5.79 & 3.35 & 0.22 \\
Model 6 & $\boldsymbol{B}_{T}$ & $2 \times 10^{-4}$ & $2 \times 10^{-4}$ & 5 & 2000 & 0.22 & 16.46 & 1.82 & 0.06 \\
Model 7 & $\boldsymbol{B}_{P}$ & $10^{-3}$ & $10^{-3}$ & 5 & 400 & 0.36 & 8.43 & 2.18 & 0.13 \\
Model 8 & $\boldsymbol{B}_{P}$ & $10^{-3}$ & $5 \times 10^{-3}$ & 25 & 400 & 1.48 & 2.77 & 1.83 & 0.37 \\
Model 9 & $\boldsymbol{B}_{P}$ & $10^{-3}$ & $2 \times 10^{-4}$ & 1 & 400 & 0.07 & 29.50 & 2.63 & 0.04 \\
Model 10 & $\boldsymbol{B}_{P}$ & $10^{-3}$ & $-10^{-3}$ & -5 & 400 & 0.31 & 7.90 & 0.70 & 0.13 \\
\hline
\end{tabular}




\section{References}

1. H. K. Moffatt, Magnetic field generation in electrically conducting fluids, ed. Cambridge (1978).

2. N. Bucciantini and L. Del Zanna, MNRAS 428, 71 (2013).

3. L. Del Zanna, O. Zanotti, N. Bucciantini and P. Londrillo, A\&SA 473, 11 (2007).

4. L. Pareschi and G. Russo, Journal of Scientific Computing 25, p. 129 (2005).

5. C. Palenzuela, L. Lehner, O. Reula and L. Rezzolla, MNRAS 394, 1727 (2009).

6. M. Gilfanov, X-Ray Emission from Black-Hole Binaries, in Lecture Notes in Physics, Berlin Springer Verlag, ed. T. Belloni, Vol. 794, p.17 (2010). 